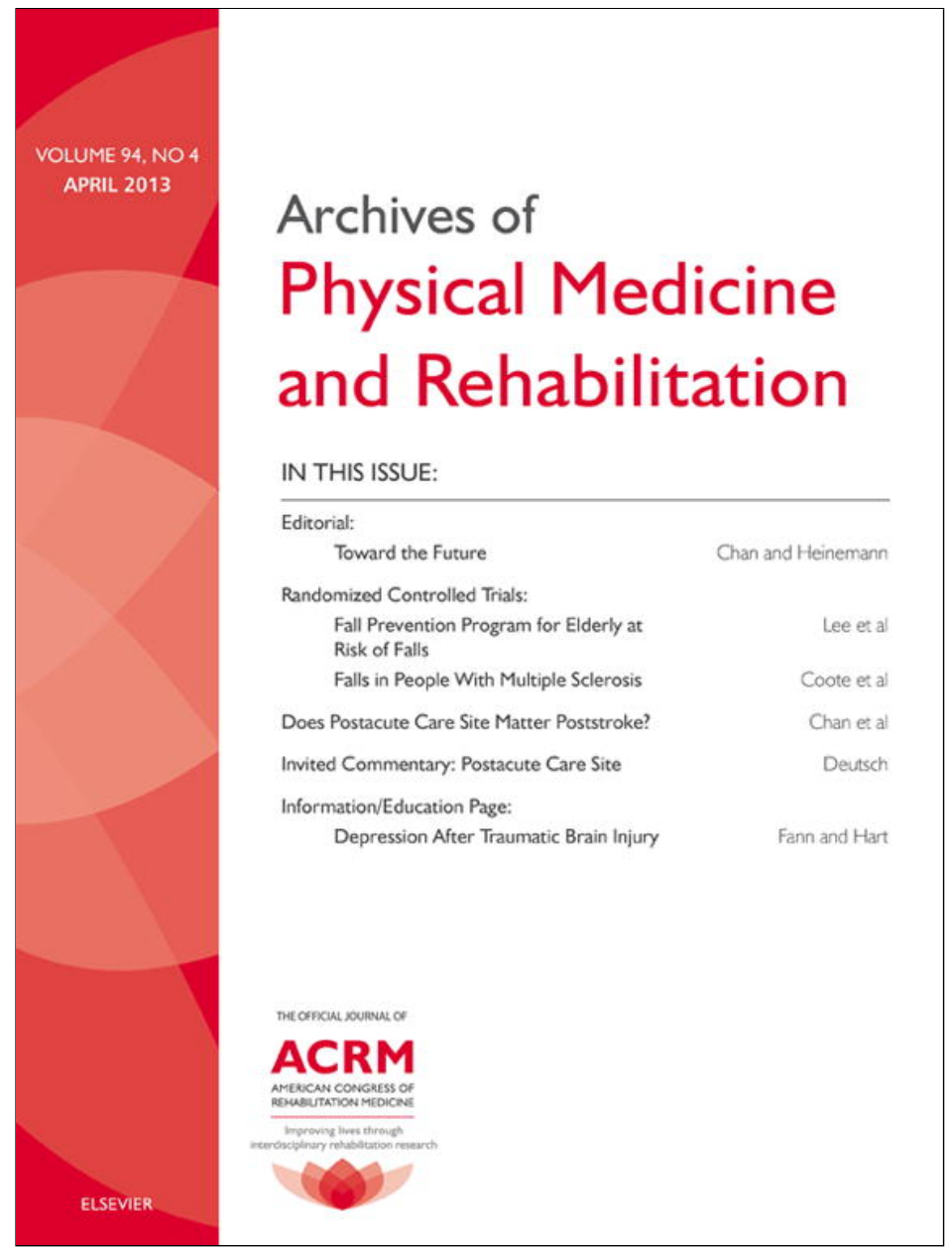

This article appeared in a journal published by Elsevier. The attached copy is furnished to the author for internal non-commercial research and education use, including for instruction at the authors institution and sharing with colleagues.

Other uses, including reproduction and distribution, or selling or licensing copies, or posting to personal, institutional or third party websites are prohibited.

In most cases authors are permitted to post their version of the article (e.g. in Word or Tex form) to their personal website or institutional repository. Authors requiring further information regarding Elsevier's archiving and manuscript policies are encouraged to visit:

http://www.elsevier.com/authorsrights 


\title{
Gait Analysis With Cognitive-Motor Dual Tasks to Distinguish Fallers From Nonfallers Among Rehabilitating Stroke Patients
}

\author{
Tina Baetens, PT, ${ }^{a}$ Alexandra De Kegel, PT, ${ }^{\mathrm{b}}$ Tanneke Palmans, Ing, ${ }^{\mathrm{b}}$ Kristine Oostra, MD, ${ }^{\mathrm{c}}$ \\ Guy Vanderstraeten, MD, PhD, ${ }^{a, c}$ Dirk Cambier, PT, PhD ${ }^{\mathrm{a}}$ \\ From the ${ }^{a}$ Department of Rehabilitation Sciences and Physiotherapy, Ghent University, Ghent; ${ }^{b}$ Department of Rehabilitation Sciences and \\ Physiotherapy, University College Artevelde, Ghent; and the ${ }^{c}$ Department of Physical and Rehabilitation Medicine, Ghent University Hospital, \\ Ghent, Belgium.
}

\begin{abstract}
Objectives: To evaluate fall risk in stroke patients based on single- and dual-task gait analyses, and to investigate the difference between 2 cognitive tasks in the dual-task paradigm.

Design: Prospective cohort study.

Setting: Rehabilitation hospitals.

Participants: Subacute stroke patients $(\mathrm{N}=32)$, able to walk without physical/manual help with or without walking aids, while performing a verbal task.

Interventions: Not applicable.

Main Outcome Measures: Functional gait measures were Functional Ambulation Categories (FAC) and use of a walking aid. Gait measures were evaluated by an electronic walkway system under single- and dual-task (DT) conditions. For the single-task, subjects were instructed to walk at their usual speed. One of the DTs was a verbal fluency dual task, whereby subjects had to walk while simultaneously enumerating as many different animals as possible. For the other DT (counting dual task), participants had to walk while performing serial subtractions. After inclusion, participants kept a 6-month falls diary. Results: Eighteen (56.3\%) of the 32 included patients fell. Ten (31.3\%) were single fallers (SFs), and 8 (25\%) were multiple fallers (MFs). Fallers (Fs) more frequently used a walking aid and more frequently needed an observatory person for walking safely (FAC score of 3) than nonfallers (NFs). Two gait decrement parameters in counting dual task could distinguish potential Fs from NFs: decrement in stride length percentage $(P=.043)$ and nonparetic step length percentage $(P=.047)$. Regarding the division in 3 groups (NFs, SFs, and MFs), only MFs had a significantly higher percentage of decrement for paretic step length $(P=.023)$ than SFs.

Conclusions: Examining the decrement of spatial gait characteristics (stride length and paretic and nonparetic step length) during a DT addressing working memory can identify fall-prone subacute stroke patients.
\end{abstract} Archives of Physical Medicine and Rehabilitation 2013;94:680-6

(c) 2013 by the American Congress of Rehabilitation Medicine

Falling, a common complication poststroke, can involve many consequences. ${ }^{1}$ Several studies demonstrated that falls in stroke survivors often occur during locomotion ${ }^{1-5}$ and gait deficits, for example, problems with obstacle-crossing or need of supervision for safety (Functional Ambulation Category $[\mathrm{FAC}]^{6}$ score of 3) are retained as an important fall-risk factor. However, different patients, all categorized with an FAC score of 3 , can still

No commercial party having a direct financial interest in the results of the research supporting this article has or will confer a benefit on the authors or on any organization with which the authors are associated. differ significantly in qualitative performance or in quantitative temporal-spatial gait characteristics. These characteristics also may fluctuate based on varying circumstances, for example, walking while simultaneously performing another task. Hyndman and Ashburn ${ }^{7}$ evaluated the ability of walking while talking, known as the "Stops walking when talking" test (SWWT) in chronic stroke patients and found it to be moderately predictive for falling. Better results were revealed for distinguishing nonrepeated from repeated fallers than nonfallers (NFs) from fallers (Fs). Andersson et $\mathrm{al}^{8}$ confirmed the test's predictive value in subacute patients. 
Walking while talking, a plain application of a cognitive-motor dual task (DT), can create destabilizing effects because of the competing demands for attention resources needed for both tasks. If 2 simultaneously performed tasks require more than the total information processing capacity, the performance on either or both deteriorates. ${ }^{9}$ Therefore, clinicians often emphasize the importance of not talking while walking, and therefore patients can turn all attention to maintaining balance.

Even so, these DTs are increasingly included in temporal-spatial gait analyses, and different studies have investigated gait-related cognitive-motor interference (CMI) in stroke patients. ${ }^{10-15}$ They found different effects on gait parameters when patients walked and simultaneously performed a cognitive task, including slower gait speed, ${ }^{12}$ reduced cadence, longer stride duration, and increased double-limb support. ${ }^{11,15}$ In a neurologic population, DT-related gait decrements showed to be significantly correlated with the patients' functional independence (Barthel Index) score, rather than with one of the standardly used gait measures, being the ten-meter walk time. ${ }^{13}$ These results can emphasize the benefit of gait analyses, including DT paradigms, besides clinical observations of gait when evaluating stroke patients.

In older adults, gait analysis with DTs are frequently used in fall assessment, ${ }^{16,17}$ and some studies provided an indication that DTs may have an added value. Different outcome parameters from DT walking indicating a fall risk were the following: reduced walking speed, ${ }^{18}$ changes in step width, step time, and step length, ${ }^{17}$ or variability in stride time. ${ }^{19}$

In chronic stroke patients, Hyndman et $\mathrm{al}^{15}$ found that Fs showed a significantly reduced stride length during DT gait analyses compared with NFs.

An important issue in studies on CMI is the nature of the secondary task, because previous research has shown that different types of secondary (cognitive) tasks have varying effects on gait parameters. ${ }^{9,10,20,21}$ In older adults, cognitive DTs that cause more competitive interaction with executive functions are preferred to provoke DT-related gait changes. ${ }^{20,21}$ In community-dwelling stroke patients, spontaneous speech, as addressed in the SWWT, produced more gait interference than working memory or visuospatial tasks. ${ }^{10}$ Hyndman ${ }^{15}$ used a silent cognitive task (remembering a shopping list), drawing on sustained and divided attention as well as short-term memory, to explore differences between Fs and NFs.

The aims of the present study were to assess differences between NFs and Fs, as well as between NFs, single fallers (SFs), and multiple fallers (MFs), based on analysis using an electronic walkway system during a solitary walk, as well as 2 DTs in the first 6 months after stroke. Based on previous studies, ${ }^{17,20} 2$ DTs were used, addressing mainly semantic (enumerating animals) or

\begin{tabular}{|ll|}
\hline List of abbreviations: \\
CMI & cognitive-motor interference \\
DT & dual task \\
F & faller \\
FAC & Functional Ambulation Categories \\
MF & multiple faller \\
MI & Motricity Index \\
MMSE & Mini-Mental State Examination \\
NF & nonfaller \\
SF & single faller \\
ST & single task \\
SWWT & "Stops walking when talking" test \\
\hline
\end{tabular}

working memory (counting backwards by threes). Two different cognitive tasks were used to investigate the importance of type of DT in gait analysis, evaluating fall risk. A final aim was to determine which gait parameters are the most important in fall evaluation and-if the DT paradigm has an additional merit - which task should be used. More insight into the effects of specific DTs on the mobility and fall risk of subacute stroke patients may be useful in future research on gait training paradigms in order to prevent stroke patients from falling.

\section{Methods}

\section{Participants}

All participants had residual hemiparesis and were recruited within the first 6 months after a first-time stroke. They had been included in an extensive study on risk factors for falling $(\mathrm{N}=73) .{ }^{5}$ For this gait analysis study, patients had to be able to walk without manual help, with or without walking assistive devices (FAC score $\geq 3$ ) and did not have aphasia or dysarthria interfering with verbal tasks $(n=38)$. Figure 1 illustrates the study design and dropouts. All patients had to be able to understand the meaning of the study and to follow instructions. The study protocol was approved by the central and local ethics committees, and all patients signed an informed consent of participation after explanation of the study and prior to data collection.

Data of 32 patients were included for analyses. The mean age was 64.8 years (range, 32-90) with no significant sex difference. Strokes were predominantly ischemic in origin $(75 \%)$, and the mean duration since stroke onset was mean \pm SD $10.6 \pm 6.7$ weeks.

\section{Design}

Patients underwent a baseline screening and were followed (on fall status) for 6 months. Personal and medical information was collected before assessments took place. Cognitive status was examined using the Mini-Mental State Examination (MMSE) ${ }^{22}$ A screening gait observation was performed whereby the use of a walking aid and the level of (physical) support in order to ambulate safely (expressed as FAC $^{6}$ ) was registered. The severity of hemiplegia was assessed using the Motricity Index (MI). ${ }^{23}$

\section{Gait analysis}

Spatial and temporal measures of gait were determined by the GaitRite system, ${ }^{\mathrm{a}}$ an electronic walkway connected to a personal computer with specific software. The GaitRite system has shown to be a valid and reliable method to evaluate spatial and temporal gait parameters. ${ }^{24-26}$ Patients had to walk independently on the GaitRite walkway (with a total length of $5.79 \mathrm{~m}$ ), starting and ending both at a marked point $2 \mathrm{~m}$ from the walkway in order to eliminate the effect of acceleration and deceleration. Patients used their customary assistive devices during all conditions. For the single task (ST), patients were instructed to walk at their normative speed. Before the verbal fluency dual task, while walking, patients received the following instructions: walk at your normative speed while simultaneously verbally enumerating as many different animals as possible, and for the counting dual task, they were told the following: walk at your normative speed while simultaneously 


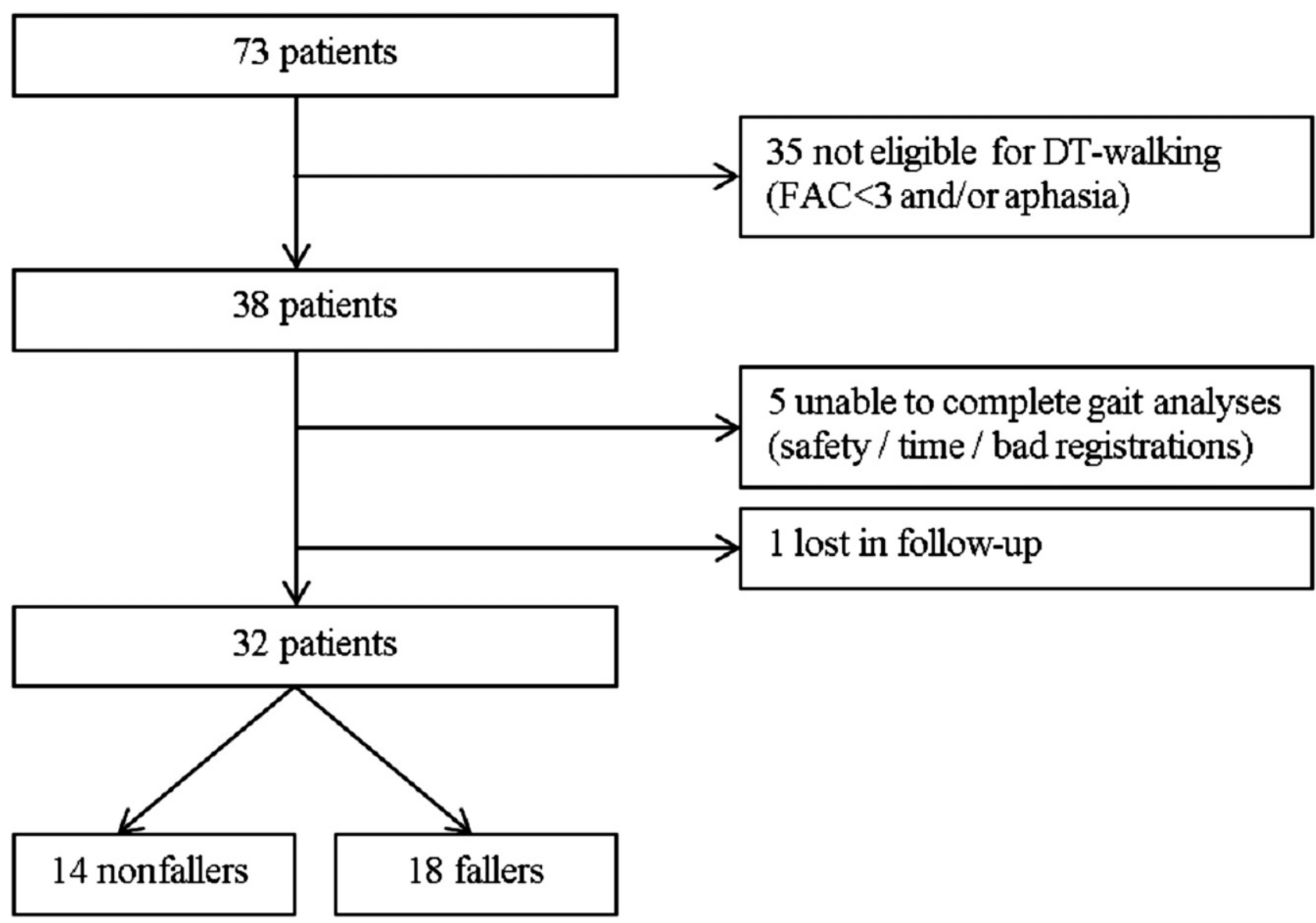

Fig 1 Number of study participants.

performing serial subtractions by threes starting from 100 and counting out loud. Before starting the DT, an instruction not to prioritize the gait or cognitive task was given, creating a condition in which attention was divided. The parameters selected from GaitRite were the following: velocity $(\mathrm{cm} / \mathrm{s})$, cadence (steps $/ \mathrm{min})$, step length at the paretic and nonparetic side $(\mathrm{cm})$, and stride length $(\mathrm{cm})$. For all these basic parameters, the DT-related gait decrement $(\%)$ was calculated, using the formula: decrement $=(\mathrm{ST}$ score-DT score)/ST score $\times 100 \%{ }^{27}$

\section{Fall registration}

After baseline assessment, all patients were followed up on falls for 6 months, using fall calendars. A fall was defined as an unexpected loss of balance resulting in coming to rest on the ground or an object below knee level, not due to a violent blow or intrinsic event, for example, fainting or an epileptic seizure. ${ }^{28}$ Every month calendars had to be sent to the principal investigator. When a fall was reported, patients were contacted to ask about the circumstances. A phone call was also made when fall calendars were not returned on time. Based on their fall status, subjects were classified into 2 groups: NFs or Fs. Additionally, Fs were classified into SFs or MFs.

\section{Data analysis}

All data were analyzed using SPSS 19.0. ${ }^{\mathrm{b}}$ Concerning patient characteristics, differences between Fs and NFs were analyzed with Pearson chi-square or Fisher exact test (categorical variables), with independent $t$ test (age/time since stroke onset/MI) or Mann-Whitney $U$ test (MSSE). To evaluate differences between NFs, SFs, and MFs, Pearson chi-square or Fisher exact test (categorical), 1-way analysis of variance (age/time since stroke onset/MI), or Kruskal-Wallis test (MMSE) were used.

For every separate gait parameter, the difference between the conditions (ST, verbal fluency dual task, counting dual task) was examined using paired sample $t$ tests. Differences in the basic gait and gait-decrement parameters between groups (based on fall status) were evaluated by analysis of variance through the general linear model. Additionally, use of a walking aid was included as a second between-subjects factor, because the use of a walking aid may influence gait parameters among ambulatory stroke patients. ${ }^{29,30}$ Bonferroni post hoc comparisons were made when required. All tests were 2-sided, and the confidence level was set at $95 \%$ with $P=.05$.

\section{Results}

\section{Participants}

Eighteen patients $(56.3 \%)$ fell during the 6-month follow-up: 10 $(31.3 \%)$ fell once, and $8(25 \%)$ fell 2 or more times. Patients' characteristics are provided in table 1 . Patients suffering a hemorrhagic or mixed stroke tended to fall more $(P=.053)$, but no significant differences in patients' characteristics were observed between NFs and Fs. Looking specifically at the Fs, no differences 
Table 1 Characteristics of participants

\begin{tabular}{lllll}
\hline \multirow{2}{*}{ Characteristics } & & \multicolumn{2}{c}{ Fs } \\
\cline { 3 - 5 } & NFs $(n=14)$ & Total $(n=18)$ & SFs $(n=10)$ & MFs $(n=8)$ \\
\hline Age $(\mathrm{y})$ & $66.4 \pm 15.5$ & $63.6 \pm 16.7$ & $63.2 \pm 15.3$ & $64.1 \pm 19.4$ \\
Young/old (>65y) & $6 / 8$ & $9 / 9$ & $5 / 5$ & $4 / 4$ \\
Sex (male/female) & $12 / 2$ & $10 / 8$ & $6 / 4$ & $4 / 4$ \\
Stroke type (ischemic/hemorrhagic) & $13 / 1$ & $11 / 7$ & $6 / 4$ & $5 / 3$ \\
Hemiplegic side (right/left) & $8 / 6$ & $8 / 10$ & $3 / 7$ & $5 / 3$ \\
Time since stroke onset (wk) & $8.1 \pm 5.2$ & $12.4 \pm 7.2$ & $10.9 \pm 5.6$ & $14.4 \pm 8.8$ \\
MMSE & $27.0(22-30)$ & $28.0(20-30)$ & $28.0(23-30)$ & $28.0(20-29)$ \\
MI & $76.0 \pm 12.5$ & $67.4 \pm 14.8$ & $65.5 \pm 15.5$ & $70.1 \pm 14.4$ \\
Use of walking aid (no/yes) & $9 / 5^{* \dagger}$ & $5 / 13^{*}$ & $2 / 8^{\dagger}$ & $3 / 5$ \\
FAC score (3/4-5) & $1 / 13^{* \dagger}$ & $8 / 10^{*}$ & $5 / 5^{\dagger}$ & $3 / 5$ \\
\hline
\end{tabular}

NOTE. Values are mean \pm SD (for independent $t$ test or 1-way analysis of variance) or number (for Pearson chi-square or Fisher exact test) or median (range) for Mann-Whitney $U$ test and Kruskal-Wallis test.

* Significant difference between NFs and Fs $(P<.05)$.

Significant difference between NFs and SFs $(P<.05)$.

in characteristics were found between SFs versus MFs, or between the 3 groups (NFs, SFs, and MFs).

More than half of all participants (56.3\%) used a walking aid at the moment of gait evaluation, with no significant differences between the different FACs. Only 3 patients were assessed as an FAC score of 5, and none of them fell. To avoid (quasi-)empty categories, FAC scores of 4 and 5 were combined in 1 functional category, as previously described. ${ }^{5}$

Comparisons between Fs and NFs showed that Fs more frequently used a walking aid $(P=.039)$ and more frequently were defined as walkers in need of supervision for safety (FAC score of 3) $(P=.044)$. No significant differences between SFs and MFs were found. Compared with the NFs group, more SFs, as well as more MFs, were classified as FAC 3 (able to walk without physical/ manual help). Less NFs used a walking aid than SFs or MFs.
Significance for these differences could only be shown between NFs and SFs. Additionally, participants' age did not differ between the different fall groups, nor were there differences between MMSE score, use of a walking aid, or FAC score between the young or older $(>65 \mathrm{y})$ patients.

\section{Quantitative gait analysis}

Comparing all 3 conditions (ST, verbal fluency dual task, counting dual task) with each other (table 2), all basic gait parameters did show significant differences between the ST and verbal fluency dual task and between ST and DT-count. Paretic step length and stride length showed significant differences between verbal fluency dual task and counting dual task. However, no significant differences

Table 2 Gait parameters during the ST walking and DT conditions

\begin{tabular}{|c|c|c|c|c|c|c|}
\hline \multirow[b]{2}{*}{ Gait Parameter } & \multirow[b]{2}{*}{ Condition } & \multirow[b]{2}{*}{ Total $(\mathrm{N}=32)$} & \multirow[b]{2}{*}{ NFs $(n=14)$} & \multicolumn{3}{|c|}{ Fs } \\
\hline & & & & Total $(n=18)$ & SFs $(n=10)$ & MFs $(n=8)$ \\
\hline \multirow[t]{3}{*}{ Cadence (steps/min) } & ST & $72.7 \pm 23.6^{*}$ & $76.5 \pm 21.7$ & $69.8 \pm 25.1$ & $70.0 \pm 24.6$ & $69.6 \pm 27.5$ \\
\hline & DT-verb & $62.9 \pm 23.8^{*}$ & $65.0 \pm 26.1$ & $61.3 \pm 22.4$ & $57.7 \pm 23.1$ & $65.8 \pm 22.3$ \\
\hline & DT-count & $62.5 \pm 24.4^{*}$ & $64.2 \pm 23.7$ & $61.3 \pm 25.5$ & $56.5 \pm 23.0$ & $67.3 \pm 28.8$ \\
\hline \multirow[t]{3}{*}{ Velocity $(\mathrm{cm} / \mathrm{s})$} & ST & $50.2 \pm 27.9^{*}$ & $57.1 \pm 29.3$ & $44.8 \pm 26.3$ & $46.1 \pm 28.1$ & $43.1 \pm 25.7$ \\
\hline & DT-verb & $40.3 \pm 24.4^{*}$ & $45.5 \pm 28.0$ & $36.3 \pm 21.2$ & $37.5 \pm 25.1$ & $34.8 \pm 16.8$ \\
\hline & DT-count & $37.7 \pm 22.4^{*}$ & $43.1 \pm 23.8$ & $33.6 \pm 21.0$ & $34.9 \pm 25.2$ & $31.8 \pm 15.7$ \\
\hline \multirow[t]{3}{*}{ Stride length $(\mathrm{cm})$} & ST & $77.7 \pm 24.7^{\dagger}$ & $85.0 \pm 28.4$ & $72.1 \pm 20.6$ & $74.0 \pm 21.1$ & $69.7 \pm 21.0$ \\
\hline & DT-verb & $71.8 \pm 23.6^{\dagger}$ & $77.6 \pm 27.1$ & $67.3 \pm 20.2$ & $71.9 \pm 20.8$ & $61.5 \pm 19.2$ \\
\hline & DT-count & $68.5 \pm 21.7^{\dagger}$ & $76.3 \pm 22.1$ & $62.4 \pm 19.9$ & $68.3 \pm 21.6$ & $55.1 \pm 15.7$ \\
\hline \multirow[t]{3}{*}{ Paretic step length $(\mathrm{cm})$} & ST & $41.5 \pm 10.7^{\dagger}$ & $44.4 \pm 12.0$ & $39.2 \pm 9.2$ & $40.8 \pm 8.4$ & $37.1 \pm 10.3$ \\
\hline & DT-verb & $38.7 \pm 11.0^{\dagger}$ & $40.9 \pm 11.8$ & $36.9 \pm 10.3$ & $39.6 \pm 9.1$ & $33.6 \pm 11.2$ \\
\hline & DT-count & $36.5 \pm 10.5^{\dagger}$ & $39.5 \pm 9.4$ & $34.2 \pm 10.9$ & $38.3 \pm 9.3$ & $29.2 \pm 11.1$ \\
\hline \multirow[t]{3}{*}{ Nonparetic step length (cm) } & ST & $36.0 \pm 15.4^{*}$ & $40.3 \pm 17.2$ & $32.7 \pm 13.4$ & $33.0 \pm 14.7$ & $32.3 \pm 12.5$ \\
\hline & DT-verb & $32.9 \pm 15.0^{*}$ & $36.6 \pm 16.4$ & $30.1 \pm 13.5$ & $32.0 \pm 15.4$ & $27.6 \pm 11.2$ \\
\hline & DT-count & $31.7 \pm 13.3^{*}$ & $36.5 \pm 13.6$ & $28.0 \pm 12.1$ & $29.8 \pm 14.9$ & $25.8 \pm 7.7$ \\
\hline
\end{tabular}

NOTE. Values are mean \pm SD.

Abbreviations: DT-count, counting dual task; DT-verb, verbal fluency dual task.

* Significant difference between ST and DT-verb and between ST and DT-count $(P<.001)$.

$\dagger$ Significant difference between all 3 conditions (ST, DT-verb, and DT-count) $(P<.05)$. 
Table 3 DT-related gait decrement

\begin{tabular}{|c|c|c|c|c|}
\hline \multirow[b]{2}{*}{ Gait Parameter } & \multirow[b]{2}{*}{ NFs $(n=14)$} & \multicolumn{3}{|c|}{ Fs } \\
\hline & & Total $(n=18)$ & SFs $(n=10)$ & MFs $(n=8)$ \\
\hline \multicolumn{5}{|l|}{ Cadence (\%) } \\
\hline DT-verb & $15.4 \pm 19.8$ & $10.9 \pm 16.5$ & $18.2 \pm 10.5$ & $1.7 \pm 18.7$ \\
\hline DT-count & $17.3 \pm 13.7$ & $12.3 \pm 21.0$ & $19.1 \pm 16.9$ & $3.9 \pm 23.6$ \\
\hline \multicolumn{5}{|l|}{ Velocity (\%) } \\
\hline DT-verb & $21.4 \pm 21.8$ & $17.0 \pm 16.9$ & $20.0 \pm 13.0$ & $13.1 \pm 21.1$ \\
\hline DT-count & $24.5 \pm 13.6$ & $23.7 \pm 21.2$ & $24.9 \pm 17.6$ & $22.2 \pm 26.3$ \\
\hline \multicolumn{5}{|c|}{ Stride length $(\%)$} \\
\hline DT-verb & $8.5 \pm 10.9$ & $6.6 \pm 9.7$ & $2.4 \pm 9.2$ & $11.8 \pm 7.9$ \\
\hline DT-count & $8.3 \pm 10.0^{* \dagger}$ & $12.8 \pm 13.4^{*}$ & $7.6 \pm 9.1^{\dagger}$ & $19.2 \pm 15.6^{\dagger}$ \\
\hline \multicolumn{5}{|c|}{ Paretic step length (\%) } \\
\hline DT-verb & $7.7 \pm 11.1$ & $6.6 \pm 9.3$ & $3.4 \pm 6.0$ & $10.6 \pm 11.5$ \\
\hline DT-count & $9.8 \pm 10.1^{\dagger}$ & $13.4 \pm 15.2$ & $6.3 \pm 7.5^{\dagger}$ & $22.3 \pm 18.2^{\dagger}$ \\
\hline \multicolumn{5}{|c|}{ Nonparetic step length (\%) } \\
\hline DT-verb & $10.2 \pm 14.1$ & $8.3 \pm 20.1$ & $3.3 \pm 25.8$ & $14.6 \pm 6.7$ \\
\hline DT-count & $5.9 \pm 16.6^{*}$ & $12.2 \pm 20.4^{*}$ & $9.3 \pm 23.5$ & $15.9 \pm 16.7$ \\
\hline
\end{tabular}

NOTE. Values are mean \pm SD.

Abbreviations: DT-count, counting dual task; DT-verb, verbal fluency dual task.

* Significant difference between NFs and Fs $(P<.05)$.

$\dagger$ Significant difference between NFs, SFs, and MFs $(P<.05)$.

in any gait parameter during the ST or DT condition was seen between groups based on their fall status (NFs and Fs and NFs, SFs, and MFs). Additionally, no significant differences in any gait parameter between fall groups were seen when taking into consideration the use of a walking aid.

The DT-related gait decrements are reported in table 3. No gait decrements regarding the verbal fluency dual task could distinguish NFs from Fs. When comparing gait decrements in counting dual task, 2 parameters differed between Fs and NFs, showing significant main effects of fall status for the gait decrement for stride length percentage $(P=.043)$ and nonparetic step length percentage $(P=.047)$. Regarding the division in 3 groups (NFs, $\mathrm{SFs}$, and MFs), a significant main effect of fall group was seen for the parameters decrement stride length percentage $(P=.022)$ and paretic step length percentage $(P=.019)$. Post hoc analyses showed that MFs had a significantly higher percentage of decrement for paretic step length $(P=.023)$ than SFs.

\section{Discussion}

This study investigated the usefulness of gait analysis in isolation and combined with a cognitive task for fall evaluation among stroke patients. It differs from previous work, because it explores this topic among subacute stroke patients-most falls occur during the first months in or after rehabilitation ${ }^{31,32}$-and it uses 2 different secondary tasks presumed to cause CMI and, to our knowledge, is not yet used as part of a fall-risk evaluation in this population.

All gait parameters during both DTs differed significantly from those obtained in the ST, which has been reported in previous research. ${ }^{13,15}$ It shows that both DTs were divergent enough to cause $\mathrm{CMI}$ in this population.

However, no significant group differences could be observed for the basic gait parameters during solitary walking as well as during the DTs. Differences could only be seen in the parameters expressing the decrement in counting dual task compared with the ST, and not for any decrement in the verbal fluency dual task. This highlights the importance of DT type for detecting gait changes related to falling in this population. The difference in the ability to distinguish NFs from Fs between the 2 cognitive tasks may be attributed to different factors, such as differences in the type of memory addressed, in the level of perceived difficulty and in the rhythmic and articulomotor component.

The verbal fluency task mainly depends on semantic memory, whereas the backward counting task depends on working memory. ${ }^{20}$ The working memory is more directly related to executive functions and is even often considered a component of executive functions. ${ }^{33,34}$ Moreover, some theoretical models consider working memory as the central component of the executive system. ${ }^{34}$ Therefore, the higher gait decrement shown in the counting dual task among Fs compared with NFs may be related to impaired executive functioning. An independent association of executive functioning with balance and mobility has already been described in chronic ${ }^{35}$ and subacute stroke patients. ${ }^{36}$ Moreover, in subacute nonambulatory right-hemisphere stroke patients, impaired executive functioning was a predictive fall-risk factor. ${ }^{36}$

A difference in rhythm between the cognitive tasks may partially provoke the gait decrement differences. It has been shown that 2 simultaneously performed rhythmic activities with different frequencies highly interfere with each other. ${ }^{37}$ The importance of (ar)rhythm of the secondary task was already shown by Beauchet et al, ${ }^{20}$ who investigated the effect of different DTs on gait among older adults, and whereby also used a verbal fluency task (enumerating animals) and an arrhythmic counting back-task. Hereby a significant increase in stride time was found when walking while performing the verbal fluency task and the counting backward task compared with solitary walking. However, a significant increase in stride time variability was only found for the arrhythmic DT. ${ }^{20}$

Talking aloud demands articulation, and therefore this articulation component itself may also explain some of the observed 
motor interference. Therefore, 2 secondary tasks requiring articulation, believed to cause more interference than a silent task, ${ }^{15}$ were chosen for the present DTs. Additionally, it has been proven that the allowance of variation in both gait and cognitive tasks provides a better representation of daily living activities. ${ }^{38}$ Speaking aloud made it possible to control whether prioritization did not occur. Although Plummer-D'Amato et $\mathrm{al}^{10}$ did show that spontaneous speech produced more gait interference than working memory or visuospatial tasks in chronic patients, spontaneous speech was not chosen as a secondary task in order to ensure a verifiable sufficient amount of utterances on a relatively short walking distance. Additionally, the possible answers for the 2 other tasks of Plummer-D' Amato et $\mathrm{al}^{10}$ were yes or no, requiring little articulation. ${ }^{10}$ Our counting dual task and verbal fluency dual task required more articulation and respiration than these yes-no responses.

The present findings support the results of Hyndman et al, ${ }^{15}$ who stated that a reduced stride length during DT walking distinguishes Fs from NFs. Moreover, it demonstrates that DT paradigms are already useful for fall evaluation early after stroke, at the beginning of independent walking, when increased mobility may increase exposure to fall opportunities as well. Additionally, our findings suggest that different spatial parameters (stride length as well as step length at the paretic and nonparetic side) can be useful in differentiation between NFs, SFs, and MFs; however, a validation on a larger sample of all subgroups is recommended.

We acknowledge the user-friendliness of the SWWT; however, as a single assessment, it showed good specificity, but very low sensitivity in predicting Fs from NFs. ${ }^{7,8}$ Therefore, many patients at high risk of falling can continue walking during this assessment; however, they do present with spatial gait decrements during DTs that may be detected with gait analysis.

The strengths of this study were the different secondary tasks, which were easy to perform in clinical practice, as well as the prospective design, and the few exclusion criteria: no age criteria were fixed, and therefore patients of different ages were included. However, this large population-like variation in age may entail a possible threat for general interpretation of DT results. It could be that CMI is because of increased age rather than health status. However, age (categories) were equally spread among Fs and NFs, and comparisons of the different characteristics as well as the gait (decrement) parameters based on age (categories) did not reveal any differences. These CMIs because of stroke, rather than age, were previously seen in studies using a cognitive verbal-response task, ${ }^{7,12}$ whereas the contrast was seen in studies using a silent mental task. ${ }^{15}$

All patients able to walk independently with or without a walking aid and with or without the need of supervision for safe walking were also included. Based on these criteria, it was possible to have a heterogeneous group representing the able-to-walk subacute stroke population, ${ }^{39}$ except for patients with aphasia or cognitive deficits, based on the spoken DT paradigm. Patients performed all walks with their customary aids, and it was demonstrated that Fs more often used an aid than NFs. Tyson and Ashburn ${ }^{29}$ did already report an association between use of an aid and severity of hemiplegia and gait performance. A prospective study among neurologic inpatients identified the use of a walking aid as a fall risk factor as well. ${ }^{40}$ The difference between walking aid use in NFs and MFs could not be statistically demonstrated, possibly because of the smaller subgroups. However, it can also be hypothesized that MFs are taking more risks and thus regularly do not use aids in spite of prescription for safety. Further research with a larger sample is necessary to clarify this matter. Our exploratory analyses showed that most basic gait parameters differed between aid users and nonusers, indicating the importance of including this factor in analysis. Especially in DT conditions, addressing divided attention, the use of a walking aid is important, because the use of an aid in itself already is attentiondemanding. ${ }^{41}$ Several studies on DT gait analysis included stroke patients with the need for a walking aid. ${ }^{10-13,15}$ Only 1 reported numbers ( 1 cane user among 8 participants). ${ }^{11}$

\section{Study limitations}

A limitation of this study was the absence of assessing the performance of the cognitive task. The number of correct responses was not registered, which could have given additional information. Hyndman et $\mathrm{al}^{15}$ did analyze the results of the cognitive task and found a significant decrease of cognitive task performance during the DT walking among stroke patients, but no differences were seen between Fs and NFs or right- or left-hemisphere stroke patients. Secondly, in spite of a representative sample of the heterogeneous able-to-walk stroke group, an enlargement of the rather small sample would have been better to generalize our results.

\section{Conclusions}

The decrement of spatial gait characteristics during a DT addressing working memory can identify fall-prone subacute stroke patients when taking into account the use of a walking aid: specific gait decrement percentages for stride length and nonparetic step length can distinguish Fs from NFs and the decrement for paretic step length percentage can distinguish MFs from SFs. The need of a walking aid as well as attention, memory, and executive function abilities during walking are important factors to consider in fall-risk evaluation. When practicing walking abilities, exercises that minimize the division of attention may result in better gait performance. However, because many patients fall during locomotion and many situations in daily life require divided attention, therapists have to be aware that it may be useful to practice DT walking in all ambulatory patients. It even may be necessary to include training of these DTs with different cognitive tasks (causing different CMI) to lower the fall risk in rehabilitating stroke patients.

\section{Suppliers}

a. CIR Systems Inc, 376 Lafayette Ave, Ste 202, Sparta, NJ 07871.

b. SPSS Inc, 233 S Wacker Dr, 11th Fl, Chicago, IL 60606.

\section{Keywords}

Accidental falls; Gait; Rehabilitation; Stroke

\section{Corresponding author}

Tina Baetens, PT, Rehabilitation Sciences and Physiotherapy Ghent, Campus Heymans (UZ) 1B3, De Pintelaan 185, BE-9000 Ghent, Belgium. E-mail address: Tina.Baetens@Ugent.be.

\section{Acknowledgments}

We thank all therapists and doctors of the participating hospitals/ rehabilitation centers (Centre for musculoskeletal and neurological 
rehabilitation of the Ghent University Hospital-Ghent, Heilig Hart hospital-Roeselare, Jan Palfijn hospital-Ghent, Rehabilitation centre De Mick-Brasschaat, and AZ Sint-Jan hospital AV-Bruges) who were willing to cooperate in the screening and follow-up.

\section{References}

1. Weerdesteyn V, de Niet M, van Duijnhoven HJ, Geurts AC. Falls in individuals with stroke. J Rehabil Res Dev 2008;45:1195-214.

2. Nyberg L, Gustafson Y. Patient falls in stroke rehabilitation. A challenge to rehabilitation strategies. Stroke 1995;26:838-42.

3. Forster A, Young J. Incidence and consequences of falls due to stroke: a systematic inquiry. BMJ 1995;311:83-6.

4. Soyuer F, Ozturk A. The effect of spasticity, sense and walking aids in falls of people after chronic stroke. Disabil Rehabil 2007;29:679-87.

5. Baetens T, De Kegel A, Calders P, Vanderstraeten G, Cambier D. Prediction of falling among stroke patients in rehabilitation. J Rehab Med 2011;43:876-83.

6. Holden MK, Gill KM, Magliozzi MR. Gait assessment for neurologically impaired patients. Standards for outcome assessment. Phys Ther 1986;66:1530-9.

7. Hyndman D, Ashburn A. 'Stops walking when talking' as a predictor of falls in people with stroke living in the community. J Neurol Neurosurg Psych 2004;75:994-7.

8. Andersson AG, Kamwendo K, Seiger A, Appelros P. How to identify potential fallers in a stroke unit: validity indexes of 4 test methods. J Rehabil Med 2006;38:186-91.

9. Woollacott M, Shumway-Cook A. Attention and the control of posture and gait: a review of an emerging area of research. Gait Posture 2002;16:1-14.

10. Plummer-D'Amato P, Altmann LJ, Saracino D, Fox E, Behrman AL, Marsiske M. Interactions between cognitive tasks and gait after stroke: a dual task study. Gait Posture 2008;27:683-8.

11. Plummer-D'Amato P, Altmann LJ, Behrman AL, Marsiske M. Interference between cognition, double-limb support, and swing during gait in community-dwelling individuals poststroke. Neurorehab Neural Repair 2010;24:542-9.

12. Bowen A, Wenman R, Mickelborough J, Foster J, Hill E, Tallis R. Dual task effects of talking while walking on velocity and balance following a stroke. Age Ageing 2001;30:319-23.

13. Haggard P, Cockburn J, Cock J, Fordham C, Wade D. Interference between gait and cognitive tasks in a rehabilitating neurological population. J Neurol Neurosurg Psych 2000;69:479-86.

14. Canning CG, Ada L, Paul SS. Is automaticity of walking regained after stroke? Disabil Rehabil 2006;28:97-102.

15. Hyndman D, Ashburn A, Yardley L, Stack E. Interference between balance, gait and cognitive task performance among people with stroke living in the community. Disabil Rehabil 2006;28:849-56.

16. Zijlstra A, Ufkes T, Skelton DA, Lundin-Olsson L, Zijlstra W. Do dual tasks have an added value over single tasks for balance assessment in fall prevention programs? A mini-review. Gerontology 2008;54:40-9.

17. Nordin E, Moe-Nilssen R, Ramnemark A, Lundin-Olsson L. Changes in step-width during dual-task walking predicts falls. Gait Posture 2010;32:92-7.

18. Beauchet O, Annweiler C, Allali G, Berrut G, Herrmann FR, Dubost V. Recurrent falls and dual task-related decrease in walking speed: is there a relationship? J Am Geriatr Soc 2008;56:1265-9.

19. Kressig RW, Herrmann FR, Grandjean R, Michel JP, Beauchet O. Gait variability while dual-tasking: fall predictor in older inpatients? Aging Clin Exp Res 2008;20:123-30.
20. Beauchet O, Dubost V, Aminian K, Gonthier R, Kressig RW. Dualtask-related gait changes in the elderly: does the type of cognitive task matter? J Mot Behav 2005;37:259-64.

21. Holtzer R, Verghese J, Xue X, Lipton RB. Cognitive processes related to gait velocity: results from the Einstein aging study. Neuropsych 2006;20:215.

22. Folstein MF, Folstein SE, McHugh PR. Mini-Mental State: a practical method for grading the cognitive state of patients for the clinician. J Psychiatr Res 1975;12:189-98.

23. Collen FM, Wade DT, Bradshaw CM. Mobility after stroke: reliability of measures of impairment and disability. Int Disabil Stud 1990;12:6-9.

24. Bilney B, Morris M, Webster K. Concurrent related validity of the GAITRite-walkway system for quantification of the spatial and temporal parameters of gait. Gait Posture 2003;17:68-74.

25. Menz HB, Latt MD, Tiedemann A, Mun San Kwan M, Lord SR. Reliability of the GAITRite-walkway system for the quantification of temporo-spatial parameters of gait in young and older people. Gait Posture 2004;20:20-5

26. Kuys SS, Brauer SG, Ada L. Test-retest reliability of the GAITRite system in people with stroke undergoing rehabilitation. Disabil Rehabil 2011;19:1848-53.

27. Yang YR, Chen YC, Lee CS, Cheng SJ, Wang RY. Dual-task-related gait changes in individuals with stroke. Gait Posture 2007;25:185-90.

28. Zecevic AA, Salmoni AW, Speechley M, Vandervoort AA. Defining a fall and reasons for falling: comparisons among the views of seniors, health care providers, and the research literature. Gerontologist 2006; 46:367-76.

29. Tyson SF, Ashburn A. The influence of walking aids on hemiplegic gait. Physiother Theory Pract 1994;10:77-86.

30. Kuan TS, Tsou JY, Su FC. Hemiplegic gait of stroke patients: the effect of using a cane. Arch Phys Med Rehabil 1999;80:777-84.

31. Suzuki T, Sonoda S, Misawa K, Saitoh E, Shimizu Y, Kotake T. Incidence and consequence of falls in inpatient rehabilitation of stroke patients. Exp Aging Res 2005;31:457-69.

32. Mackintosh SF, Hill K, Dodd KJ, Goldie P, Culham E. Falls and injury prevention should be part of every stroke rehabilitation plan. Clin Rehabil 2005;19:441-51.

33. Anderson V, Jacobs R, Anderson PJ. Executive functions and the frontal lobes: a lifespan perspective. New York: Psychology Pr; 2008.

34. Baddeley A. Working memory. Science 1992;255:556-9.

35. Liu-Ambrose T, Pang MY, Eng JJ. Executive function is independently associated with performances of balance and mobility in communitydwelling older adults after mild stroke: implications for falls prevention. Cerebrovasc Dis 2006;23:203-10.

36. Rapport LJ, Webster JS, Flemming KL, et al. Predictors of falls among right-hemisphere stroke patients in the rehabilitation setting. Arch Phys Med Rehabil 1993;74:621-6.

37. Taga G, Yamaguchi Y, Shimizu H. Self-organized control of bipedal locomotion by neural oscillators in unpredictable environment. Biol Cybern 1991;65:147-59.

38. Verghese J, Kuslansky G, Holtzer R, et al. Walking while talking: effect of task prioritization in the elderly. Arch Phys Med Rehabil 2007;88:50-3.

39. Jorgensen HS, Nakayama H, Raaschou HO, Olsen TS. Recovery of walking function in stroke patients: the Copenhagen Stroke Study. Arch Phys Med Rehabil 1995;76:27-32.

40. Stolze H, Klebe S, Zechlin C, Baecker C, Friege L, Deuschl G. Falls in frequent neurological diseases. J Neurol 2004;251:79-84.

41. Wright DL, Kemp TL. The dual-task methodology and assessing the attentional demands of ambulation with walking devices. Phys Ther 1992;72:306-12. 